\title{
Periodic and Non-Periodic Solutions of a Ricker-type Second-Order Equation with Periodic Parameters ${ }^{1}$
}

\author{
N. LAZARYAN and H. SEDAGHAT 2
}

\begin{abstract}
We study the dynamics of the positive solutions of the exponential difference equation

$$
x_{n+1}=x_{n-1} e^{a_{n}-x_{n-1}-x_{n}}
$$

where the sequence $\left\{a_{n}\right\}$ is periodic. We find that qualitatively different dynamics occurs depending on whether the period $p$ of $\left\{a_{n}\right\}$ is odd or even. If $p$ is odd then periodic and nonperiodic solutions coexist (with different initial values) if the amplitudes of the terms $a_{n}$ are allowed to vary over a sufficiently large range. But if $p$ is even then all solutions converge to an asymptotically stable limit cycle of period $p$ if either all the odd-indexed or all the even-indexed terms of $\left\{a_{n}\right\}$ are less than 2 , and the sum of the even terms of $\left\{a_{n}\right\}$ does not equal the sum of its odd terms. The key idea in this analysis is a semiconjugate factorization of the above equation into a triangular system of two first-order equations.
\end{abstract}

\section{Introduction}

We study the behavior of solutions of the second-order difference equation

$$
x_{n+1}=x_{n-1} e^{a_{n}-x_{n-1}-x_{n}}
$$

where the parameter $\left\{a_{n}\right\}$ is a periodic sequence of real numbers. This equation is a special case of a stage-structured population model with a Ricker-type recruitment function; see [3]. For more information and additional Ricker-type models see, e.g. [5], 6], 7] and [11.

Equation (1) has a rich variety of periodic and non-periodic solutions. It exhibits coexisting periodic solutions if the range of variation, or amplitude of $a_{n}$ is limited. We also show that an expanded range or greater amplitudes for $a_{n}$ leads to the occurrence of coexisting non-periodic solutions (including chaotic solutions) as well.

In addition, there is an unexpected qualitative difference between the behavior of solutions when the period $p$ of $\left\{a_{n}\right\}$ is odd and when $p$ is even. When $p$ is odd different periodic and non-periodic stable solutions are generated from different pairs of initial values; see Corollary 9 and Theorem 13 below. But if $p$ is even and the amplitude of $a_{n}$ is less than 2 then Theorem 18 shows that asymptotically stable $p$-cycles occur in a generic fashion, i.e. independently of initial values.

\footnotetext{
${ }^{1}$ Journal of Difference Equations and Applications, DOI 10.1080/10236198.2016.1187142

${ }^{2}$ Department of Mathematics, Virginia Commonwealth University, Richmond, VA 23284, USA;

Email: hsedagha@vcu.edu
} 
Such differences in behavior among the solutions of (1) are readily explained by a semiconjugate factorization of (1) into a triangular system of two first-order difference equations. The latter pair of equations determine the full structure of (1) and allow us to explain the aforementioned variety of behaviors that its solutions exhibit as follows: One of the two first-order equations has periodic, hence bounded solutions when $p$ is odd; see Lemma 5 . However, if $p$ is even then the solutions of the same first-order equation are unbounded (except for a boundary case); see Lemma 14 and the remarks following it. Thus the corresponding bounded solutions of (1) "forget" the initial values and approach a single asymptotically stable solution.

The paper is divided into sections that highlight the natural dichotomy that exists between odd and even periods. Detailed calculations that appear as a sequence of lemmas are necessary to prove the aforementioned statements. A careful review of the lemmas may be deferred until after Corollary 9, Theorem 13 and Theorem 18 have been examined along with the remarks and figures that illustrate their statements.

\section{Order reduction}

It is convenient to note that if the initial values $x_{-1}, x_{0}$ are positive then $x_{n}>0$ for all $n \geq 1$ and

$$
x_{n+1}<x_{n-1} e^{a_{n}-x_{n-1}} \leq e^{a_{n}} \frac{1}{e}=e^{a_{n}-1}
$$

Thus the following result is obvious.

Lemma 1 Let $\left\{a_{n}\right\}$ be a sequence of real numbers that is bounded from above with $\sup _{n} a_{n}=a$. If $x_{-1}, x_{0}>0$ then the corresponding solution $\left\{x_{n}\right\}$ of 11 is bounded and for all $n$

$$
0<x_{n}<e^{a-1} \text {. }
$$

The study of (1) is facilitated by the fact that it admits a semiconjugate factorization that decomposes it into two equations of order one. Following [9], we define

$$
t_{n}=\frac{x_{n}}{x_{n-1} e^{-x_{n-1}}}
$$

for each $n \geq 1$ and note that

$$
t_{n+1} t_{n}=\frac{x_{n+1}}{x_{n} e^{-x_{n}}} \frac{x_{n}}{x_{n-1} e^{-x_{n-1}}}=\frac{x_{n+1}}{x_{n-1} e^{-x_{n-1}-x_{n}}}=e^{a_{n}}
$$

or equivalently,

$$
t_{n+1}=\frac{e^{a_{n}}}{t_{n}} .
$$


Now

$$
x_{n+1}=e^{a_{n}} x_{n-1} e^{-x_{n-1}} e^{-x_{n}}=e^{a_{n}} \frac{x_{n}}{t_{n}} e^{-x_{n}}=\frac{e^{a_{n}}}{t_{n}} x_{n} e^{-x_{n}}=t_{n+1} x_{n} e^{-x_{n}}
$$

The pair of equations (3) and (4) constitute the semiconjugate factorization of (1):

$$
\begin{aligned}
t_{n+1} & =\frac{e^{a_{n}}}{t_{n}}, \quad t_{0}=\frac{x_{0}}{x_{-1} e^{-x_{-1}}} \\
x_{n+1} & =t_{n+1} x_{n} e^{-x_{n}}
\end{aligned}
$$

Every solution $\left\{x_{n}\right\}$ of (1) is generated by a solution of the system (5)-(6). Using the initial values $x_{-1}, x_{0}$ we obtain a solution $\left\{t_{n}\right\}$ of the first-order equation (5), called the factor equation. This solution is then used to obtain a solution of the cofactor equation (6) and thus also of (1). The system (5)-(6) is said to be triangular basically because one equation (i.e. the factor equation) is independent of the other; see [10] for more information on triangular systems.

For an arbitrary sequence $\left\{a_{n}\right\}$ and a given $t_{0} \neq 0$ by iterating (5) we obtain

$$
t_{1}=\frac{e^{a_{0}}}{t_{0}}, \quad t_{2}=\frac{e^{a_{1}}}{t_{1}}=t_{0} e^{-a_{0}+a_{1}}, \quad t_{3}=\frac{e^{a_{2}}}{t_{2}}=\frac{1}{t_{0}} e^{a_{0}-a_{1}+a_{2}}, \quad t_{4}=\frac{e^{a_{3}}}{t_{3}}=t_{0} e^{-a_{0}+a_{1}-a_{2}+a_{3}}, \cdots
$$

This pattern of development implies the following result.

Lemma 2 Let $\left\{a_{n}\right\}$ be an arbitrary sequence of real numbers and $t_{0} \neq 0$.

(a) The general solution of (5) is given by

$$
t_{n}=t_{0}^{(-1)^{n}} e^{(-1)^{n} s_{n}}, \quad n=1,2, \ldots
$$

where

$$
s_{n}=\sum_{j=1}^{n}(-1)^{j} a_{j-1}
$$

(b) For all n,

$$
x_{n} \leq \frac{1}{e} t_{n}
$$

Proof. (a) For $n=1$, (7) yields

$$
t_{1}=t_{0}^{-1} e^{-s_{1}}=\frac{1}{t_{0}} e^{-\left(-a_{0}\right)}=\frac{e^{a_{0}}}{t_{0}}
$$

which is true. Suppose that (7) is true for $n \leq k$. Then by (7) and (8)

$$
t_{0}^{(-1)^{k+1}} e^{(-1)^{k+1} s_{k+1}}=\frac{1}{t_{0}^{(-1)^{k}} e^{(-1)^{k} s_{k}}} e^{(-1)^{2 k+2} a_{k}}=\frac{e^{a_{k}}}{t_{k}}=t_{k+1}
$$


which is again true and the proof is now complete by induction.

(b) This is an immediate consequence of (6) and the fact that $x e^{-x} \leq 1 / e$.

In the sequel, whenever the sequence $\left\{a_{n}\right\}$ has period $p$ the following quantity plays an essential role:

$$
\sigma=s_{p}=\sum_{j=1}^{p}(-1)^{j} a_{j-1}=-a_{0}+a_{1}-a_{2}+\ldots-a_{p-1}
$$

The following special-case result will be useful later on.

Lemma 3 Assume that $\left\{a_{n}\right\}$ is periodic with minimal period $p$. If $\sigma=0$ and $t_{0}=1$, then $\left\{t_{n}\right\}$ is periodic with period $p$.

Proof. If $\sigma=0$, then by (7) and (8) in Lemma 2 we have:

$$
t_{p}=t_{0}^{(-1)^{p}} e^{(-1)^{p} s_{p}}=e^{(-1)^{p} \sigma}=1=t_{0}
$$

and

$$
t_{n+p}=t_{0}^{(-1)^{n+p}} e^{(-1)^{n+p} s_{n+p}}=e^{(-1)^{n+p} s_{n+p}} .
$$

Now since $\sigma=0$,

$$
s_{n+p}=\sum_{j=1}^{n+p}(-1)^{j} a_{j-1}=\sum_{j=1}^{p}(-1)^{j} a_{j-1}+\sum_{j=p+1}^{p+n}(-1)^{j} a_{j-1}=\sum_{j=p+1}^{p+n}(-1)^{j} a_{j-1}
$$

If $p$ is even, then

$$
\sum_{j=p+1}^{p+n}(-1)^{j} a_{j-1}=-a_{p}+a_{p+1}+\cdots+(-1)^{n+p} a_{n+p-1}=-a_{0}+a_{1}+\cdots+(-1)^{n} a_{n-1}=s_{n}
$$

so

$$
t_{n+p}=e^{(-1)^{n+p} s_{n+p}}=e^{(-1)^{n} s_{n}}=t_{n} .
$$

If $p$ is odd, then

$$
\sum_{j=p+1}^{p+n}(-1)^{j} a_{j-1}=a_{p}-a_{p+1}+\cdots+(-1)^{n+p} a_{n+p-1}=a_{0}-a_{1}+\cdots-(-1)^{n} a_{n-1}=-s_{n}
$$

So

$$
t_{n+p}=e^{(-1)^{n+p} s_{n+p}}=e^{-(-1)^{n}\left(-s_{n}\right)}=e^{(-1)^{n} s_{n}}=t_{n} .
$$

and the proof is complete.

Note that the solution $\left\{t_{n}\right\}$ of (5) in Lemma 2 need not be bounded even if $\left\{a_{n}\right\}$ is a bounded sequence. The next result expresses a useful fact for this case. 
Lemma 4 Assume that $\left\{a_{n}\right\}$ is bounded from above and $x_{0}, x_{-1}>0$. If the sequence $\left\{t_{n}\right\}$ from $t_{0}$ given in (5) is unbounded then some subsequence of the corresponding solution $\left\{x_{n}\right\}$ of (1) converges to 0 .

Proof. By the hypotheses, $\sup _{n} a_{n}=a<\infty$ and there is a subsequence $\left\{t_{n_{k}}\right\}$ such that $\lim _{k \rightarrow \infty} t_{n_{k}}=\infty$. By (5) and Lemma 2(b)

$$
x_{n_{k}+1} \leq \frac{1}{e} t_{n_{k}+1} \leq \frac{e^{a-1}}{t_{n_{k}}}
$$

Therefore,

$$
\lim _{k \rightarrow \infty} x_{n_{k}+1}=\lim _{k \rightarrow \infty} \frac{e^{a-1}}{t_{n_{k}}}=0 .
$$

\section{The odd period case}

The dynamics of (1) depends critically on whether the period of the parameter sequence $\left\{a_{n}\right\}$ is odd or even. In this section we consider the odd case and the nature of solutions of (3) in this case.

Lemma 5 Suppose that $\left\{a_{n}\right\}$ is sequence of real numbers with minimal odd period $p \geq 1$ and let $\left\{t_{n}\right\}$ be a solution of (3).

(a) $\left\{t_{n}\right\}$ has period $2 p$ with a complete cycle $\left\{t_{0}, t_{1}, \ldots, t_{2 p-1}\right\}$ where $t_{k}$ is given by (7) with

$$
s_{k}=\left\{\begin{array}{l}
\sum_{j=1}^{k}(-1)^{j} a_{j-1}, \quad \text { if } 1 \leq k \leq p \\
\sum_{j=k}^{2 p-1}(-1)^{j} a_{j-p}, \quad \text { if } p+1 \leq k \leq 2 p-1
\end{array}\right.
$$

(b) If $t_{0}=e^{-\sigma / 2}$ then $\left\{t_{n}\right\}$ is periodic with period $p$.

Proof. (a) Let $\left\{a_{0}, a_{1}, \ldots, a_{p-1}\right\}$ be a full cycle of $a_{n}$ and define $\sigma$ as in (9), i.e.

$$
\sigma=-a_{0}+a_{1}-a_{2}+\ldots-a_{p-1}
$$

Since a full cycle of $a_{n}$ has an odd number of terms, expanding $s_{n}$ in (8) yields a sequence with alternating signs in terms of $\sigma$

$$
s_{n}=\sigma-\sigma+\cdots+(-1)^{m-1} \sigma+(-1)^{m} \sum_{j=1}^{i}(-1)^{j} a_{j-1}
$$


for integers $i, m$ such that $n=p m+i, m \geq 0$ and $1 \leq i \leq p$. If $m$ is even then for $i=1,2, \ldots, p$

$$
s_{n}=\sum_{j=1}^{i}(-1)^{j} a_{j-1}=\left\{\begin{array}{lll}
-a_{0} & n=p m+1 & \text { (odd) } \\
-a_{0}+a_{1} & n=p m+2 & \text { (even) } \\
\vdots & \vdots \\
-a_{0}+a_{1} \ldots-a_{p-1} & n=p m+p & \text { (odd) }
\end{array}\right.
$$

Similarly, if $m$ is odd then for $i=1,2, \ldots, p$

$$
s_{n}=\sigma-\sum_{j=0}^{i}(-1)^{j} a_{j}=\left\{\begin{array}{lll}
\sigma+a_{0} & n=p m+1 & \text { (even) } \\
\sigma+a_{0}-a_{1} & n=p m+2 & \text { (odd) } \\
\vdots & \vdots \\
\sigma+a_{0}-a_{1}+\ldots-a_{p-1} & n=p m+p & \text { (even) }
\end{array}\right.
$$

The above list repeats for every consecutive pair of values of $m$ and yields a complete cycle for $\left\{s_{n}\right\}$. In particular, for $m=0$ we obtain for $i=1,2, \ldots, p$

$$
s_{n}=\sum_{j=1}^{i}(-1)^{j} a_{j-1}= \begin{cases}-a_{0} & n=1 \\ -a_{0}+a_{1} & n=2 \\ \vdots & \vdots \\ -a_{0}+a_{1} \ldots-a_{p-1} & n=p\end{cases}
$$

and for $m=1$ we obtain for $i=1,2, \ldots, p-1$

$$
\begin{aligned}
s_{n} & =\sigma-\sum_{j=0}^{i}(-1)^{j} a_{j}= \begin{cases}a_{1}-a_{2}+\ldots-a_{p-1} & n=p+1 \\
-a_{2}+\ldots-a_{p-1} & n=p+2 \\
\vdots & \vdots \\
-a_{p-1} & n=2 p-1\end{cases} \\
& =\sum_{j=p+1}^{2 p-1}(-1)^{j} a_{j-p}
\end{aligned}
$$

This proves the validity of 10$)$ and shows that the sequence $\left\{s_{n}\right\}$ has period $2 p$. Now (7) implies that $\left\{t_{n}\right\}$ also has period $2 p$ as claimed.

(b) If $\sigma=0$ then the statement follows immediately from Lemma 3 . If $\sigma \neq 0$ and $p$ is odd then

$$
t_{p}=t_{0}^{(-1)^{p}} e^{(-1)^{p} s_{p}}=e^{\sigma / 2-\sigma}=e^{-\sigma / 2}=t_{0}
$$

In the proof of Lemma 3 it was shown that $s_{n+p}=\sigma-s_{n}$. Thus

$$
\begin{aligned}
t_{n+p} & =t_{0}^{(-1)^{n+p}} e^{(-1)^{n+p} s_{n+p}} \\
& \left.=e^{(-1)^{n} \sigma / 2} e^{-(-1)^{n}\left(\sigma-s_{n}\right.}\right) \\
& =e^{-(-1)^{n} \sigma / 2+(-1)^{n} s_{n}}=t_{0}^{(-1)^{n}} e^{(-1)^{n} s_{n}}=t_{n}
\end{aligned}
$$


and the proof is complete.

For $p=1$, Lemma 5 implies that $\left\{t_{n}\right\}$ is the two-cycle

$$
\left\{t_{0}, \frac{e^{a}}{t_{0}}\right\}
$$

where $a$ is the constant value of the sequence $\left\{a_{n}\right\}$. For $p=3,\left\{t_{n}\right\}$ is the six-cycle

$$
\left\{t_{0}, \frac{e^{a_{0}}}{t_{0}}, t_{0} e^{a_{1}-a_{0}}, \frac{e^{a_{2}-a_{1}+a_{0}}}{t_{0}}, t_{0} e^{a_{1}-a_{2}}, \frac{e^{a_{2}}}{t_{0}}\right\}
$$

From the cofactor equation (6) we obtain

$$
\begin{aligned}
& x_{2 n+2}=t_{2 n+2} x_{2 n+1} e^{-x_{2 n+1}}=t_{2 n+2} t_{2 n+1} x_{2 n} \exp \left(-x_{2 n}-t_{2 n+1} x_{2 n} e^{-x_{2 n}}\right) \\
& x_{2 n+1}=t_{2 n+1} x_{2 n} e^{-x_{2 n}}=t_{2 n+1} t_{2 n} x_{2 n-1} \exp \left(-x_{2 n-1}-t_{2 n} x_{2 n-1} e^{-x_{2 n-1}}\right)
\end{aligned}
$$

For every solution $\left\{t_{n}\right\}$ of (5), $t_{n+1} t_{n}=e^{a_{n}}$ for all $n$, so the even terms of the sequence $\left\{x_{n}\right\}$ satisfy

$$
x_{2 n+2}=x_{2 n} \exp \left(a_{2 n+1}-x_{2 n}-t_{2 n+1} x_{2 n} e^{-x_{2 n}}\right)
$$

and the odd terms satisfy

$$
x_{2 n+1}=x_{2 n-1} \exp \left(a_{2 n}-x_{2 n-1}-t_{2 n} x_{2 n-1} e^{-x_{2 n-1}}\right)
$$

To reduce the notational clutter, let

$$
y_{n}=x_{2 n} \quad \rho_{n}=a_{2 n+1} \quad \mu_{n}=t_{2 n+1}
$$

for $n \geq 0$ and also

$$
z_{n}=x_{2 n-1} \quad \zeta_{n}=a_{2 n} \quad \eta_{n}=t_{2 n} .
$$

Then we can write (11) and $(12)$ as

$$
\begin{aligned}
& y_{n+1}=y_{n} e^{\rho_{n}-y_{n}-\mu_{n} y_{n} e^{-y_{n}}} \\
& z_{n+1}=z_{n} e^{\zeta_{n}-z_{n}-\eta_{n} z_{n} e^{-z_{n}}}
\end{aligned}
$$

The next result establishes the existence of an attracting, invariant interval for (11) and (12), or equivalently, (15) and (16). 
Lemma 6 Let $\left\{a_{n}\right\}$ be a bounded sequence where $\inf _{n \geq 0} a_{n} \in(0,2)$. Let $x_{0}, x_{-1}>0$ and $t_{0}$ be given as in 55. Assume that the sequence $\left\{t_{2 n+1}\right\}$ (respectively, $\left\{t_{2 n}\right\}$ ) is bounded and let $\left\{x_{n}\right\}$ be the corresponding solution of (1).

(a) There exists an interval $[\alpha, \beta]$ with $\alpha>0$ such that if $x_{-1}, x_{0} \in[\alpha, \beta]$ then $x_{2 n} \in[\alpha, \beta]$ (respectively, $x_{2 n+1} \in[\alpha, \beta]$ ) for $n \geq 1$.

(b) For all $x_{0}, x_{-1}>0$ there exists an integer $N>0$ such that $x_{2 n} \in[\alpha, \beta]$ (respectively, $\left.x_{2 n+1} \in[\alpha, \beta]\right)$ for all $n \geq N$.

Proof. (a) First, note that if $x_{0}, x_{-1}>0$ then $x_{n}>0$ for all $n$ and by Lemma $1 x_{n} \leq e^{a-1}$ for $n \geq 1$ where

$$
a=\sup _{n \geq 0} a_{n}
$$

Thus if

$$
\beta=e^{a-1}
$$

then $x_{n} \leq \beta$ for all $n$. Next, let

$$
\rho=\inf _{n \geq 0} a_{n} \in(0,2)
$$

and consider the map

$$
f(x)=x e^{\rho-x-\gamma x e^{-x}}
$$

where $\gamma>0$ is fixed. Now $x^{*}$ is a fixed point of $f$ if and only if

$$
x^{*}=f\left(x^{*}\right)=x^{*} e^{\rho-x^{*}-\gamma x^{*} e^{-x^{*}}}
$$

which is true if and only if $\rho-x^{*}-\gamma x^{*} e^{-x^{*}}=h\left(x^{*}\right)=0$. Since $h(0)=\rho>0$ and $h(\rho)=-\gamma \rho e^{-\rho}<0$, there is $x^{*} \in(0, \rho)$ such that $h\left(x^{*}\right)=0$. Thus $f$ has a fixed point $x^{*} \in(0, \rho)$. Further $f(x)>x$ for $x \in\left(0, x^{*}\right)$ and $f(x)<x$ for $x \in\left(x^{*}, \beta\right)$. If

$$
\alpha=\min \left\{x^{*}, f(\beta), f(1)\right\} .
$$

then we now show that $[\alpha, \beta]$ is invariant under $f$, i.e. $f(x) \in[\alpha, \beta]$ for all $x \in[\alpha, \beta]$. There are two possible cases:

Case 1: $\gamma \leq e$. In this case, $f(x)$ has one critical point at $x=1$ and it is increasing in $(0,1)$ and decreasing on $(1, \infty)$. Thus $f(1)$ is a global maximum and thus $\alpha \neq f(1)$. First, consider the case where $x^{*}<f(\beta)<\beta$ and let $x \in\left[x^{*}, \beta\right]$. If $x<1$, then $f(x)>f\left(x^{*}\right)=x^{*} \geq \alpha$ because $f$ is increasing on $(0,1)$. If $x>1$, then $f(x)>f(\beta)>x^{*} \geq \alpha$, because $f$ is decreasing on $(1, \beta)$. In either case $f(x) \in[\alpha, \beta]$.

Next, consider the case where $f(\beta)<x^{*}<\beta$ and let $x \in[f(\beta), \beta]$. Then $f(x)>x>f(\beta)=\alpha$ for $f(\beta)<x<x^{*}$. On the other hand, if $x^{*}<x<1$, then $f(x)>f\left(x^{*}\right)>f(\beta)=\alpha$ and if $x^{*}<1<x<\beta$, then $f(x)>f(\beta)=\alpha$. It follows that $f(x) \in[\alpha, \beta]$ if $\gamma \leq e$. 
Case 2: $\gamma>e$. In this case, $f(x)$ has three critical points $x^{\prime}, 1$ and $x^{\prime \prime}$ with $x^{\prime}<1<x^{\prime \prime}$, where local maxima occur at $x^{\prime}$ and $x^{\prime \prime}$ and a local minimum at 1 . There are three possibilities:

(i) $\alpha=x^{*}$. In this case, for $x^{*} \leq x \leq x^{\prime}, f(x) \geq f\left(x^{*}\right)=x^{*}=\alpha$, since $f$ is increasing on $\left(0, x^{\prime}\right)$. If $x \in\left(x^{\prime}, x^{\prime \prime}\right)$, then $f(x) \geq f(1) \geq \alpha$. If $\beta \geq x^{\prime \prime}$ and $x^{\prime \prime} \leq x \leq \beta$ then $f(x) \geq f(\beta) \geq \alpha$, since $f$ is decreasing on $\left(x^{\prime \prime}, \infty\right)$.

(ii) $\alpha=f(\beta)$. In this case, for $x \in\left[f(\beta), x^{*}\right), f(x)>x \geq f(\beta)=\alpha$. If $x^{*} \leq x^{\prime}$ and $x \in\left[x^{*}, x^{\prime}\right]$ then $f(x) \geq f\left(x^{*}\right)=x^{*} \geq \alpha$ since $f$ is increasing. If $x \in\left(x^{\prime}, x^{\prime \prime}\right)$ then $f(x) \geq f(1) \geq \alpha$. If $\beta \geq x^{\prime \prime}$ and $x^{\prime \prime} \leq x \leq \beta$ then $f(x) \geq f(\beta)=\alpha$ since $f$ is decreasing.

(iii) $\alpha=f(1)$. In this case, if $x \in\left[f(1), x^{*}\right)$ then $f(x)>x>f(1)=\alpha$. If $x^{*}<x^{\prime}$ and $x \in\left[x^{*}, 1\right]$ then $f(x) \geq f\left(x^{*}\right)=x^{*} \geq \alpha$ for $x \in\left[x^{*}, x^{\prime}\right)$ and $f(x) \geq f(1)=\alpha$ for $x \in\left[x^{\prime}, 1\right]$. On the other hand, if $x^{*} \geq x^{\prime}$ then $f(x) \geq f(1)=\alpha$ for $x \in\left[x^{*}, 1\right]$. Finally, if $\beta>1$ and $x \in(1, \beta]$ then $f(x)>f(1)=\alpha$ for $x \in\left(1, x^{\prime \prime}\right)$ since $f$ is increasing on $\left(1, x^{\prime \prime}\right)$, and $f(x) \geq f(\beta) \geq \alpha$ for $x \in\left(x^{\prime \prime}, \beta\right]$ if $\beta>x^{\prime \prime}$.

The above three cases exhaust all possibilities so $f(x) \in[\alpha, \beta]$ if $\gamma>e$.

Next, assume that $\left\{t_{2 n+1}\right\}$ is bounded and let $\left\{y_{n}\right\}$ be as defined by 115 . If

$$
\gamma=\sup \left\{t_{2 n+1}\right\}+1<\infty
$$

and $y_{n} \in[\alpha, \beta]$ then

$$
y_{n+1}=y_{n} e^{a_{2 n+1}-y_{n}-t_{2 n+1} y_{n} e^{-y_{n}}}>y_{n} e^{\rho-y_{n}-\gamma y_{n} e^{-y_{n}}}=f\left(y_{n}\right) \geq \alpha
$$

Similarly, if $\left\{t_{2 n}\right\}$ is bounded and $\gamma=\sup \left\{t_{2 n}\right\}+1$ then

$$
z_{n+1}=z_{n} e^{a_{2 n+2}-z_{n}-t_{2 n+2} z_{n} e^{-z_{n}}}>z_{n} e^{\rho-z_{n}-\gamma z_{n} e^{-z_{n}}}=f\left(z_{n}\right) \geq \alpha
$$

which proves (a).

(b) It suffices to consider the case where $z_{n}, y_{n}<\alpha$. We will do this for $z_{n}$, since the case for $y_{n}$ can be done similarly. Let

$$
\tau=\sup \left\{t_{2 n}\right\}+\frac{1}{2}=\gamma-\frac{1}{2}>0
$$

so for $x<x^{*}$ and $n \geq 0$

$$
e^{a_{2 n}-x-t_{2 n} x e^{-x}}>e^{\rho-x-\tau x e^{-x}}>e^{\rho-x^{*}-\tau x^{*} e^{-x^{*}}}>e^{\rho-x^{*}-\gamma x^{*} e^{-x^{*}}}=1
$$

Define

$$
k=e^{\rho-x^{*}-\tau x^{*} e^{-x^{*}}}>1 .
$$

If $z_{n}<\alpha \leq x^{*}$, then

$$
z_{n+1}=z_{n} e^{a_{2 n+2}-z_{n}-t_{2 n+2} z_{n} e^{-z_{n}}}>z_{n} e^{\rho-x^{*}-\tau x^{*} e^{-x^{*}}}=k z_{n}
$$


If $z_{n+1}>\alpha$ then we're done; otherwise,

$$
z_{n+2}=z_{n+1} e^{a_{2 n+4}-z_{n+1}-t_{2 n+4} z_{n+1} e^{-z_{n+1}}}>z_{n+1} e^{\rho-x^{*}-t x^{*} e^{-x^{*}}}=k z_{n+1}=k^{2} z_{n}
$$

and we continue in this way inductively. Since $k>1$ it follows that $z_{n+N}>z_{n} k^{N}>\alpha$ for sufficiently large $N$.

Lemma 7 Let $\left\{a_{n}\right\}$ be periodic of period $p$ and $0<a_{n}<2$. If as noted above, $\left\{y_{n}\right\}$ and $\left\{z_{n}\right\}$ are the even and odd indexed terms of the solution $\left\{x_{n}\right\}$ of (1) with initial values $x_{0}, x_{-1} \in[\alpha, \beta]$ then there are constants $K>0$ and $\delta \in(0,1)$ such that

$$
\left|\prod_{i=0}^{n-1}\left(1-y_{i}\right)\left(1-z_{i}\right)\right| \leq K \delta^{n}
$$

Proof. Recall that if $g$ is a continuous function on the compact interval $[\alpha, \beta]$ with $|g(x)|<1$ for all $x \in[\alpha, \beta]$ then by the extreme value theorem there is a point $\tilde{x} \in[\alpha, \beta]$ such that $|g(x)| \leq|g(\tilde{x})|<1$ for $x \in[\alpha, \beta]$. Thus if $\delta=|g(\tilde{x})| \in(0,1)$ then $|g(x)| \leq \delta$ for all $x \in[\alpha, \beta]$.

Now we establish the inequality in (17). First, if $a=\max _{0 \leq i \leq p-1}\left\{a_{i}\right\}<1+\ln 2$ then $\beta=$ $e^{a-1}<2$. Thus if $u_{i}$ denotes either $y_{i}$ or $z_{i}$ then $u_{i} \in(0,2) \supset[\alpha, \beta]$, i.e. $\left|1-u_{i}\right|<1$ and there exists $\delta_{1} \in(0,1)$ so that $\left|1-u_{i}\right|<\delta_{1}$ for $u_{i} \in[\alpha, \beta]$.

Next, suppose that $a \geq 1+\ln 2$ and let

$$
2 \leq u_{i} \leq e
$$

Consider the preimage $u_{i-1}$ of $u_{i}$. There are two possible cases: Either $u_{i-1} \leq 1$ or $u_{i-1} \geq 1$.

Case 1: If $u_{i-1} \leq 1$ then

$$
\begin{aligned}
\left|1-u_{i-1}\right|\left|1-u_{i}\right| & =\left(1-u_{i-1}\right)\left(u_{i}-1\right) \\
& \leq\left(1-u_{i-1}\right)\left(u_{i-1} e^{a-u_{i-1}-\tau_{i-1} u_{i-1} e^{-u_{i-1}}}-1\right) \\
& <\left(1-u_{i-1}\right)\left(u_{i-1} e^{2-u_{i-1}}-1\right)
\end{aligned}
$$

where $\tau_{i}=\mu_{i}$ or $\eta_{i}$ depending on the case $\left(y_{n}\right.$ or $z_{n}$ respectively). Note that

$$
(1-x)\left(x e^{2-x}-1\right)<1
$$

for $x \in(0,1]$ because $(18)$ can be written as $x(1-x)<(2-x) e^{x-2}$ and this inequality is true since its left hand side has a maximum of $1 / 4$ on $(0,1]$ whereas its right hand side has a minimum of $2 e^{-2}>1 / 4$ on $(0,1]$. In particular, 18 holds for $x \in[\alpha, 1]$ so there exists $\delta_{2} \in(0,1)$ such that

$$
\left|1-u_{i-1}\right|\left|1-u_{i}\right|<\delta_{2}
$$


Case 2: If $u_{i-1} \geq 1$ then

$$
\begin{aligned}
\left|1-u_{i-1}\right|\left|1-u_{i}\right| & =\left(u_{i-1}-1\right)\left(u_{i}-1\right) \\
& \leq\left(u_{i-1}-1\right)\left(u_{i-1} e^{a-u_{i-1}-\tau_{i-1} u_{i-1} e^{-u_{i-1}}}-1\right) \\
& <\left(u_{i-1}-1\right)\left(u_{i-1} e^{a-u_{i-1}}-1\right) .
\end{aligned}
$$

If $\phi(x)=(x-1)\left(x e^{2-x}-1\right)$ then

$$
\phi^{\prime}(x)=\left[x-(x-1)^{2}\right] e^{2-x}-1, \quad \phi^{\prime \prime}(x)=(x-1)(x-4) e^{2-x} .
$$

Since $\phi$ is smooth with $\phi^{\prime}(2)=0$ and $\phi^{\prime \prime}(x)<0$ for $x \in(1,4)$ it follows that $\phi$ is maximized on $[1,4]$ at 2 and $\phi(2)=1$. In particular, for $u_{i-1} \in[1, \beta] \subset[1,4]$,

$$
\left(u_{i-1}-1\right)\left(u_{i-1} e^{a-u_{i-1}}-1\right)<\phi\left(u_{i-1}\right) \leq 1
$$

and it follows that there is $\delta_{3} \in(0,1)$ such that

$$
\left|1-u_{i-1}\right|\left|1-u_{i}\right|<\delta_{3}
$$

Finally, there are at most $m$ pairings $\left|1-u_{i-1}\right|\left|1-u_{i}\right|$ where $m=[n / 2]$ (i.e. $m$ is $n / 2$ rounded down to the nearest integer). If $n$ is even, then $m=n / 2$, if $n$ is odd, $m=(n-1) / 2$ and we have one last unpaired term left, namely, $\left|1-u_{0}\right|<(e-1)$. Choosing $\delta=\max \left\{\delta_{1}, \delta_{2}, \delta_{3}\right\}$, we get

$$
\left|\prod_{i=0}^{n-1}\left(1-u_{i}\right)\right|<(e-1) \delta^{m} .
$$

Therefore,

$$
\left|\prod_{i=0}^{n-1}\left(1-y_{i}\right)\left(1-z_{i}\right)\right|<(e-1)^{2} \delta^{2 m} \leq \frac{\beta}{\alpha} K \delta^{n m}
$$

where $K=(e-1)^{2} / \delta$ and the proof is complete.

The next result generalizes similar results in [2] and [3].

Theorem 8 Let $\left\{a_{n}\right\}$ be a periodic sequence with $0<a_{n}<2, x_{0}, x_{-1}>0$ and the sequence $\left\{t_{n}\right\}$ with $t_{0}=x_{0} / x_{-1} e^{x_{-1}}$ be periodic with period $q$. Then each solution of (1) from the initial values $x_{0}, x_{-1}$ converges to a periodic solution (dependent on the choice of initial values) with period $q$.

Proof. Let $q$ be the period of the sequence $\left\{t_{n}\right\}$ from initial value $t_{0}=\frac{x_{0}}{x_{-} e^{x}-1}$. For each $i=1,2, \cdots, q$, define the map

$$
g_{i}(x)=t_{i} x e^{-x}
$$


and let

$$
\phi=g_{q} \circ g_{q-1} \circ \cdots \circ g_{1}
$$

Then by the cofactor equation (5), $\phi$ generates the orbit of (1) from initial values $x_{0}, x_{-1}$. Also note that $\phi$ is an autonomous interval map, and by Lemma 6, there exist real numbers $\alpha, \beta>0$ and a positive integer $N$ so that $\phi:[\alpha, \beta] \rightarrow[\alpha, \beta]$ and $\phi^{n}(x) \in[\alpha, \beta]$ for all $n \geq N$. Hence, by Brouwer's fixed point theorem, there exists a $x^{*} \in[\alpha, \beta]$ so that $\phi\left(x^{*}\right)=x^{*}$. Now, let $x_{0} \in[\alpha, \beta]$ be given.

Since

$$
g_{i}^{\prime}(x)=t_{i} e^{-x}(1-x)=\frac{g_{i}(x)}{x}(1-x)
$$

then

$$
\begin{aligned}
\phi^{\prime}\left(x_{0}\right) & =\prod_{i=1}^{q} g_{i}^{\prime}\left(x_{i-1}\right)=\prod_{i=1}^{q} \frac{g_{i}\left(x_{i-1}\right)}{x_{i-1}}\left(1-x_{i-1}\right) \\
& =\frac{g_{1}\left(x_{0}\right)}{x_{0}} \frac{g_{2}\left(x_{1}\right)}{x_{1}} \ldots \frac{g_{q}\left(x_{q-1}\right)}{x_{q-1}} \prod_{i=1}^{q}\left(1-x_{i}\right)
\end{aligned}
$$

Noting that $g_{i}\left(x_{i-1}\right)=x_{i}$, we get

$$
\phi^{\prime}\left(x_{0}\right)=\frac{x_{q}}{x_{0}} \prod_{i=1}^{q}\left(1-x_{i-1}\right)
$$

Similarly,

$$
\left(\phi^{2}\right)^{\prime}\left(x_{0}\right)=(\phi \circ \phi)^{\prime}\left(x_{0}\right)=\frac{x_{2 q}}{x_{0}} \prod_{i=1}^{2 q}\left(1-x_{i-1}\right)
$$

and in general,

$$
\left(\phi^{n}\right)^{\prime}\left(x_{0}\right)=\frac{x_{n q}}{x_{0}} \prod_{i=1}^{n q}\left(1-x_{i-1}\right)
$$

Now, let $m=[n q / 2]$. If $n q$ is even, then $m=n q / 2$ and by Lemma (7)

$$
\left|\prod_{i=1}^{n q}\left(1-x_{i}\right)\right|=\left|\prod_{i=1}^{m}\left(1-y_{i}\right)\left(1-z_{i}\right)\right| \leq K \delta^{m}
$$

for some $K>0, \delta \in(0,1)$, where $y_{i}$ and $z_{i}$ are the even and odd indexed terms of the $\left\{x_{n}\right\}$ as noted above. If $n q$ is odd, then $m=(n q-1) / 2$, so

$$
\left|\prod_{i=1}^{n q}\left(1-x_{i}\right)\right|=\left|\left(1-x_{n q}\right) \prod_{i=1}^{m}\left(1-y_{i}\right)\left(1-z_{i}\right)\right| \leq(e-1) K \delta^{m}
$$


hence,

$$
\left|\left(\phi^{n}\right)^{\prime}\left(x_{0}\right)\right| \leq \frac{\alpha}{\beta}(e-1) K \delta^{(n q-1) / 2} .
$$

Finally,

$$
\left|\phi^{n}\left(x_{0}\right)-x^{*}\right|=\left|\phi^{n}\left(x_{0}\right)-\phi^{n}\left(x^{*}\right)\right|=\left|\left(\phi^{n}\right)^{\prime}(w)\right|\left|x_{0}-x^{*}\right| \leq \frac{\beta}{\alpha} K(e-1) \delta^{(n q-1) / 2}\left|x_{0}-x^{*}\right| \rightarrow 0
$$

as $n \rightarrow \infty$ and the proof is complete.

The following result on the existence of periodic solutions is a consequence of Theorem 8 .

Corollary 9 Let $\left\{a_{n}\right\}$ be periodic with minimal odd period $p$ and further assume that $0<a_{i}<2$ for $i=0, \cdots, p-1$.

(a) Each solution of (1) converges to a cycle with length $2 p$ that depends on the initial values $x_{-1}, x_{0}>0$

(b) If $x_{0}=x_{-1} e^{-\sigma / 2-x_{-1}}$ (i.e. $\left.t_{0}=e^{-\sigma / 2}\right)$, then the solutions of (1) converge to a cycle of length $p$.

Proof. (a) By Lemma 5 $\left\{t_{n}\right\}$ is periodic with period $2 p$, so $\left\{a_{n}\right\}$ and $\left\{t_{n}\right\}$ have a common period $2 p$. The rest follows from Theorem 8 .

(b) If $t_{0}=e^{-\sigma / 2}$, then by Lemma $5 t_{n}$ is periodic with period $p$. Therefore, $\left\{a_{n}\right\}$ and $\left\{t_{n}\right\}$ have a common period $p$ and the rest follows from Theorem 8 .

Remark 10 (Multistability) The periodic solutions in Corollary 9 may be distinct if the initial values are distinct, since the solutions of equations (15) and (16) depend on the sequences $\left\{t_{2 n+1}\right\}$ and $\left\{t_{2 n}\right\}$ which in turn depend on $t_{0}=x_{0} /\left(x_{-1} e^{-x_{-1}}\right)$. Thus the cycles in Corollary 9 are not locally stable, hence not ordinary limit cycles. Since cycles with different values of $t_{0}$ coexist, we see that (1) exhibits multistability. Figures 1 and 2 illustrate this situation for period $p=3$ with

$$
a_{0}=1, \quad a_{1}=1.9, \quad a_{2}=0.8 .
$$

Next, we consider a wider range of values for $a_{n}$ and the existence of non-periodic solutions for (1). The next two results are needed.

Lemma 11 Let $\left\{x_{n}\right\}$ be a solution of (1) with initial values $x_{-1}, x_{0}>0$ and assume that $\left\{a_{n}\right\}$ is periodic with minimal period $p \geq 1$ and $\left\{t_{n}\right\}$ is periodic with period $q \geq 1$. Define

$$
g_{k}(x)=t_{k} x e^{-x}, k=0,1, \ldots, q-1
$$




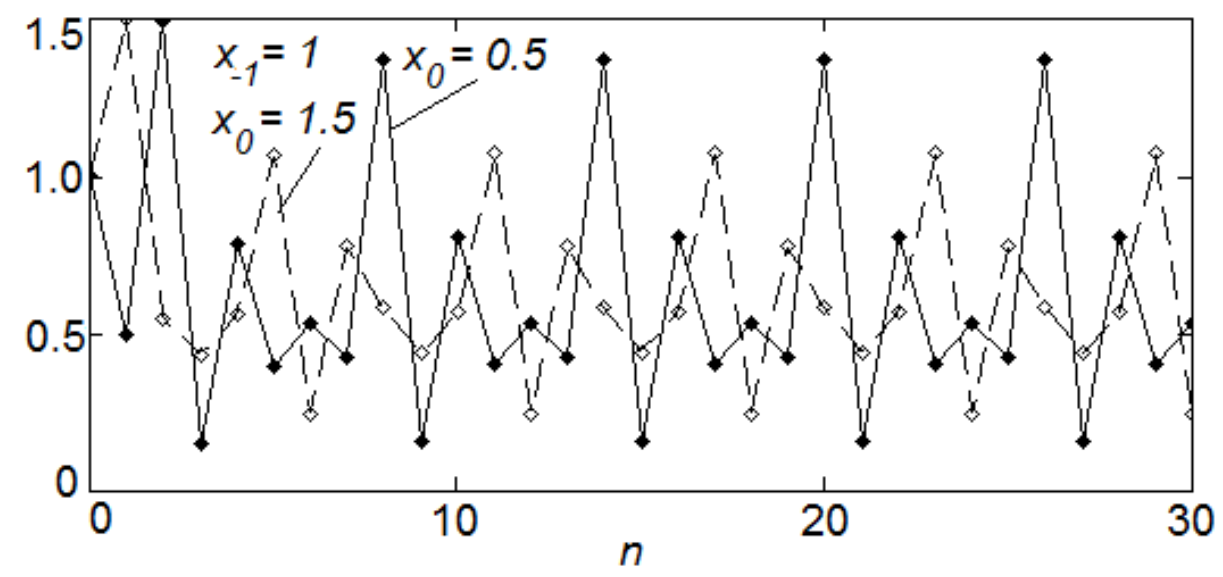

Figure 1: Coexisting period 6 solutions with parameter period $\mathrm{p}=3$

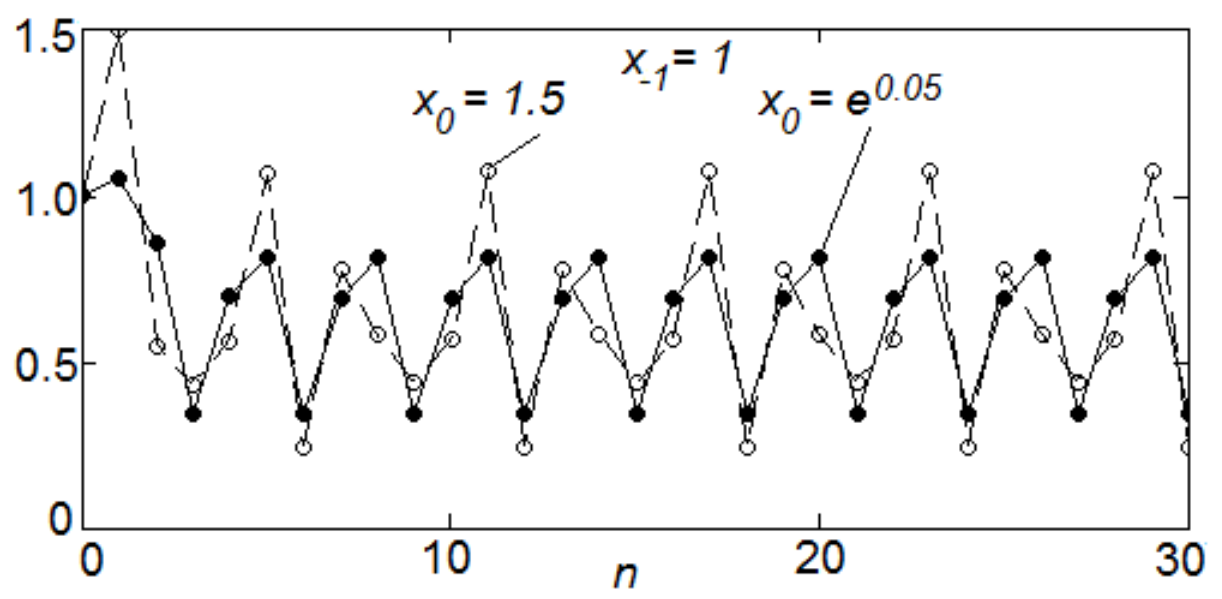

Figure 2: Coexisting period 6 and period 3 (exceptional) solutions, $\mathrm{p}=3$ 
where $t_{0}=x_{0} /\left(x_{-1} e^{-x_{-1}}\right)$ and $t_{k}$ is given by (7) and (8). Also define

$$
\begin{aligned}
h_{k} & =g_{k} \circ g_{k-1} \circ \cdots \circ g_{0}, \quad k=0,1, \ldots, q-1 \\
f & =h_{q-1}=g_{q-1} \circ g_{q-2} \circ \cdots \circ g_{1} \circ g_{0}
\end{aligned}
$$

Then $\left\{x_{n}\right\}$ is determined by the $q$ sequences

$$
x_{q m+k}=h_{k} \circ f^{m}\left(x_{-1}\right), \quad k=0,1, \ldots, q-1
$$

that are obtained by iterations of one-dimensional maps of the interval $(0, \infty)$, with $f^{0}$ being the identity map.

Proof. Given the initial values $x_{-1}, x_{0}>0$ the definition of $t_{0}$ and (6) imply that

$$
\begin{aligned}
& x_{0}=t_{0} x_{-1} e^{-x_{-1}}=g_{0}\left(x_{-1}\right)=h_{0}\left(x_{-1}\right) \\
& x_{1}=t_{1} x_{0} e^{-x_{0}}=g_{1}\left(x_{0}\right)=g_{1} \circ g_{0}\left(x_{-1}\right)=h_{1}\left(x_{-1}\right)
\end{aligned}
$$

and so on:

$$
x_{k}=h_{k}\left(x_{-1}\right), \quad k=0,1, \ldots, q-2
$$

Thus (19) holds for $m=0$. Further, $x_{q-1}=h_{q-1}\left(x_{-1}\right)=f\left(x_{-1}\right)$. Inductively, we suppose that (19) holds for some $m \geq 0$ and note that for $k=0,1, \ldots, q-2$

$$
h_{k+1}=g_{k+1} \circ g_{k} \circ \cdots \circ g_{0}=g_{k+1} \circ h_{k}
$$

Now by (6)

$$
\begin{aligned}
x_{q(m+1)-1} & =t_{q m+q-1} x_{q m+q-2} e^{-x_{q m+q-2}} \\
& =t_{q-1} h_{q-2} \circ f^{m}\left(x_{-1}\right) e^{-h_{q-2} \circ f^{m}\left(x_{-1}\right)} \\
& =g_{q-1} \circ h_{q-2} \circ f^{m}\left(x_{-1}\right) \\
& =h_{q-1} \circ f^{m}\left(x_{-1}\right) \\
& =f^{m+1}\left(x_{-1}\right)
\end{aligned}
$$

So (19) holds for $k=q-1$ by induction. Further, again by (6) and the preceding equality

$$
\begin{aligned}
x_{q(m+1)} & =t_{q m+q} x_{q m+q-1} q^{-x_{q m+q-1}} \\
& =t_{0} f^{m+1}\left(x_{-1}\right) e^{-f^{m+1}\left(x_{-1}\right)} \\
& =g_{0} \circ f^{m+1}\left(x_{-1}\right) \\
& =h_{0} \circ f^{m+1}\left(x_{-1}\right)
\end{aligned}
$$


Similarly,

$$
\begin{aligned}
x_{q(m+1)+1} & =t_{q(m+1)+1} x_{q(m+1)} e^{-x_{q(m+1)}} \\
& =t_{1} h_{0} \circ f^{m+1}\left(x_{-1}\right) e^{-h_{0} \circ f^{m+1}\left(x_{-1}\right)} \\
& =g_{1} \circ h_{0} \circ f^{m+1}\left(x_{-1}\right) \\
& =h_{1} \circ f^{m+1}\left(x_{-1}\right)
\end{aligned}
$$

Repeating this calculation $q-2$ times establishes $(19)$ and completes the induction step and the proof.

Lemma 12 Suppose that $\left\{a_{n}\right\}$ and $\left\{t_{n}\right\}$ are periodic and $\left\{t_{n}\right\}$ has minimal period $q \geq 1$.

(a) If the map $f$ in Lemma 11 has a (positive) periodic point of minimal period $\omega$ then there is a solution of (1) with period $\omega q$.

(b) If the map $f$ in Lemma 11 has a non-periodic point then (1) has a non-periodic solution.

Proof. (a) By hypothesis, there is a number $s \in(0, \infty)$ such that $f^{n+\omega}(s)=f^{n}(s)$ for all $n \geq 0$. Let $x_{-1}=s$ and define $x_{0}=h_{0}(s)$. By Lemma 11 the solution $x_{n}$ corresponding to these initial values follows the track shown below:

$$
\begin{aligned}
& x_{-1}=s \rightarrow \quad x_{0}=h_{0}(s) \rightarrow \cdots \rightarrow \quad x_{q-2}=h_{q-2}(s) \rightarrow \\
& \rightarrow x_{q-1}=h_{q-1}(s)=f(s) \rightarrow \quad x_{q}=h_{0}(f(s)) \rightarrow \cdots \rightarrow \quad x_{2 q-2}=h_{q-2}(f(s)) \rightarrow \\
& \rightarrow x_{2 q-1}=h_{q-1}(f(s))=f^{2}(s) \rightarrow x_{2 q}=h_{0}\left(f^{2}(s)\right) \rightarrow \cdots \rightarrow \quad x_{3 q-2}=h_{3 q-2}\left(f^{2}(s)\right) \rightarrow \\
& x_{\omega q-1}=h_{q-1}\left(f^{\omega-1}(s)\right)=f^{\omega}(s)=s \rightarrow \quad x_{q \omega}=h_{0}(s) \rightarrow \cdots \rightarrow \quad x_{(\omega+1) q-2}=h_{q-2}(s) \rightarrow \cdots
\end{aligned}
$$

The pattern in this list evidently repeats after $\omega q$ entries. So $x_{\omega q+n}=x_{n}$ for $n \geq 0$ and it follows that the solution $\left\{x_{n}\right\}$ of (1) has period $\omega q$.

(b) Suppose that $\left\{f^{n}\left(x_{-1}\right)\right\}$ is a non-periodic sequence for some $x_{-1}>0$. Then by Lemma 11 the solution $\left\{x_{n}\right\}$ of (1) with initial values $x_{-1}$ and $x_{0}=g_{0}\left(x_{-1}\right)$ has the non-periodic subsequence

$$
x_{q n-1}=f^{n}\left(x_{-1}\right)
$$

It follows that $\left\{x_{n}\right\}$ is non-periodic.

The following involves a wider range of values for $a_{n}$ than Corollary 9 which in particular, allows for the existence of non-periodic solutions.

Theorem 13 Suppose that $\left\{a_{n}\right\}$ is periodic with minimal odd period $p \geq 1$ and let $f$ be the interval map in Lemma 11 where $t_{0}>0$ is a fixed real number and $t_{k}$ is given by (7)-(8) for $k \geq 1$. 
(a) If $s$ is a periodic point of $f$ with period $\omega$ then all solutions of (1) with initial values $x_{-1}=s$ and $x_{0}=t_{0} s e^{-s}$ (i.e. $\left(x_{-1}, x_{0}\right)$ is on the curve $\left.g_{0}\right)$ have period $2 p \omega$.

(b) If $t_{0}=e^{-\sigma / 2}$ and $s$ is a periodic point of $f$ with period $\omega$, then all solutions of (1) with initial values $x_{-1}=s$ and $x_{0}=s e^{-\sigma / 2-s}$ have period $p \omega$ (in this case the graph of $g_{0}$ is an invariant set of (1) in the state-plane).

(c) If the map $f$ has a non-periodic point, then (1) has a non-periodic solution.

(d) If $f$ has a period-three point then (1) has periodic solutions of period $2 p n$ for all positive integers $n$ as well as chaotic solutions in the sense of Li-Yorke ([1], [4]).

Proof. Parts (a) and (c) are immediate consequences of Lemma 12 with $q=2 p$ because of Lemma 5(a). Part (b) is true by Lemma 5 (b).

(d) As is well-known from [4], if $f$ has a period three point then $f$ has periodic points of every period $n \geq 1$, as well as aperiodic, chaotic solutions in the sense of Li and Yorke. Therefore, by parts (a) and (b), (1) also has periodic solutions of period $2 p n$, as well as chaotic solutions.

In the case $p=1$, i.e. when (1) is autonomous with $a_{n}=a$ for all $n$, the conditions stated in Theorem 13 were examined in [3]. In particular, it was verified that if $a \geq 3.13$ then (1) has chaotic solutions from certain initial conditions. The multistable nature of solutions of (1) was also discussed in detail.

For odd $p \geq 3$ the map $f$ is a composition of $2 p$ functions and therefore, analytically less tractable. We use numerical simulations to highlight the rich variety of coexisting solutions that Theorem 13 allows. As noted above, and explained in greater detail in [3], these solutions are attracting (though not locally stable) so they are observable and may be recorded numerically.

In the next four figures, $p=3$ with

$$
a_{0}=1, \quad a_{1}=2, \quad a_{2}=4 .
$$

The initial values that generate the 6 -cycle in Figure 3 are $x_{-1}=1$ and $x_{0}=0.8$. Panel (a) shows the map $f=g_{5} \circ \cdots \circ g_{0}$ which is a composition of six exponential maps, together with a single stable positive fixed point that corresponds to the solution of (1) shown in Panel (b).

In Figure 4 the initial values are $x_{-1}=x_{0}=1$. In Panel (a) the graphs of $f$ and $f^{2}$ are shown that indicate the presence of a stable 2-cycle (the fixed point of $f$ is unstable in this case). Panel (b) shows the corresponding 12-cycle for (1), as required by Theorem 13 with $\omega=2$. We emphasize that this solution coexists stably with the 6 -cycle in 3 . We further note 12-cycles do not exist under the hypotheses of Corollary 9 since those hypotheses imply the stability of the fixed point of $f$.

In Figure 5 the initial values are $x_{-1}=1$ and $x_{0}=3.8$. In Panel (a) the graphs of $f$ and $f^{3}$ are shown where a stable 3 -cycle is indicated that corresponds to the solution of (1) that is shown in Panel (b). As stated in Theorem 13 this is an 18-cycle since now $\omega=3$. This solution coexists stably with the 6-cycle and the 12-cycle above. Also note that 18-cycles do not exist under the hypotheses of Corollary 9. 

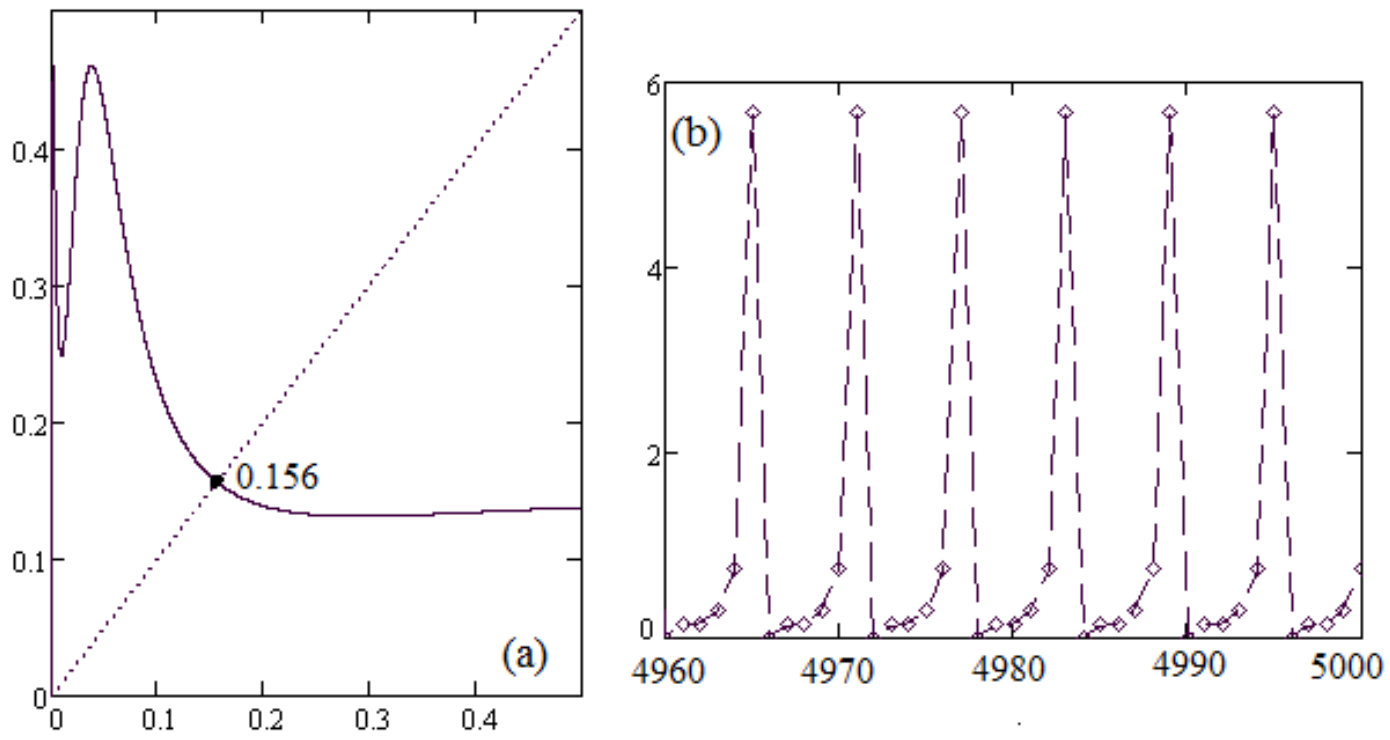

Figure 3: (a) Graph of $f$; (b) Corresponding 6-cycle
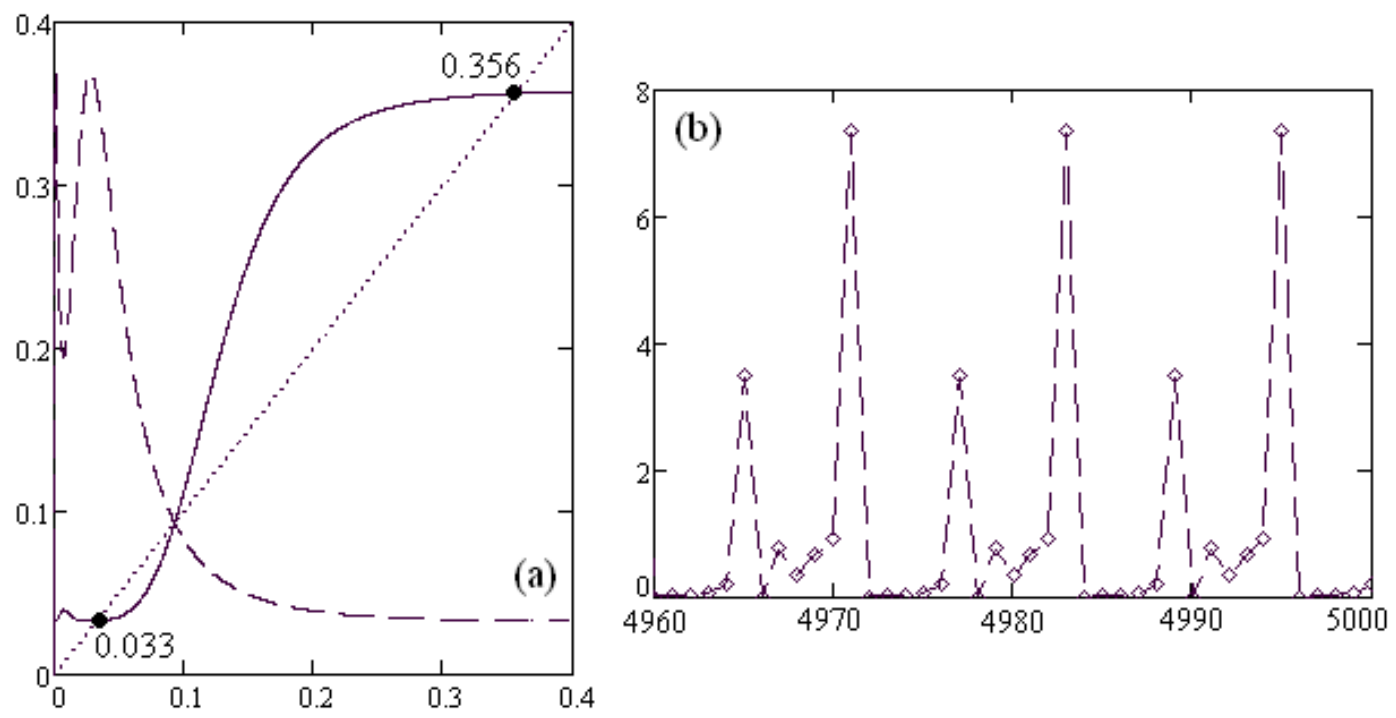

Figure 4: (a) Graphs of $f$ and $f^{2}$; (b) Corresponding 12-cycle 


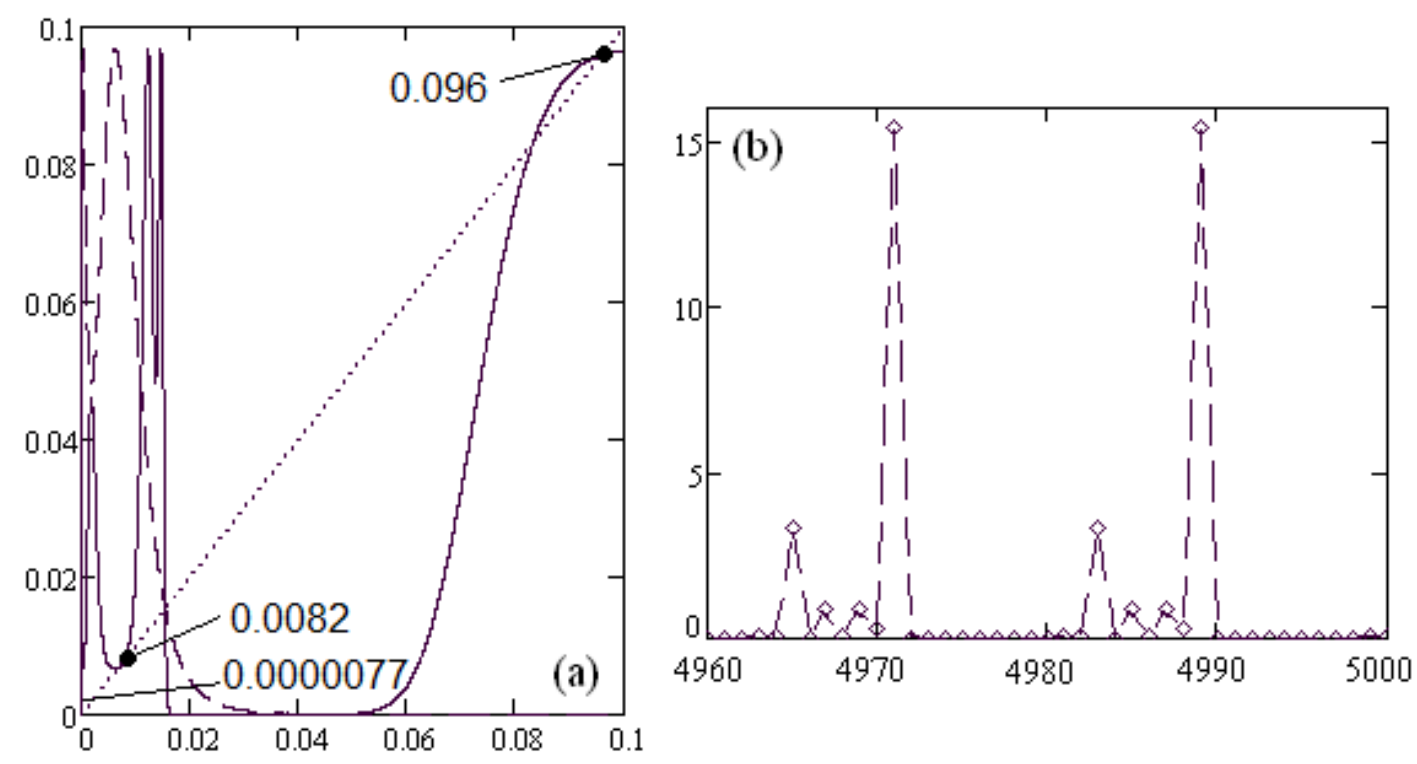

Figure 5: (a) Graphs of $f$ and $f^{3}$; (b) Corresponding 18-cycle
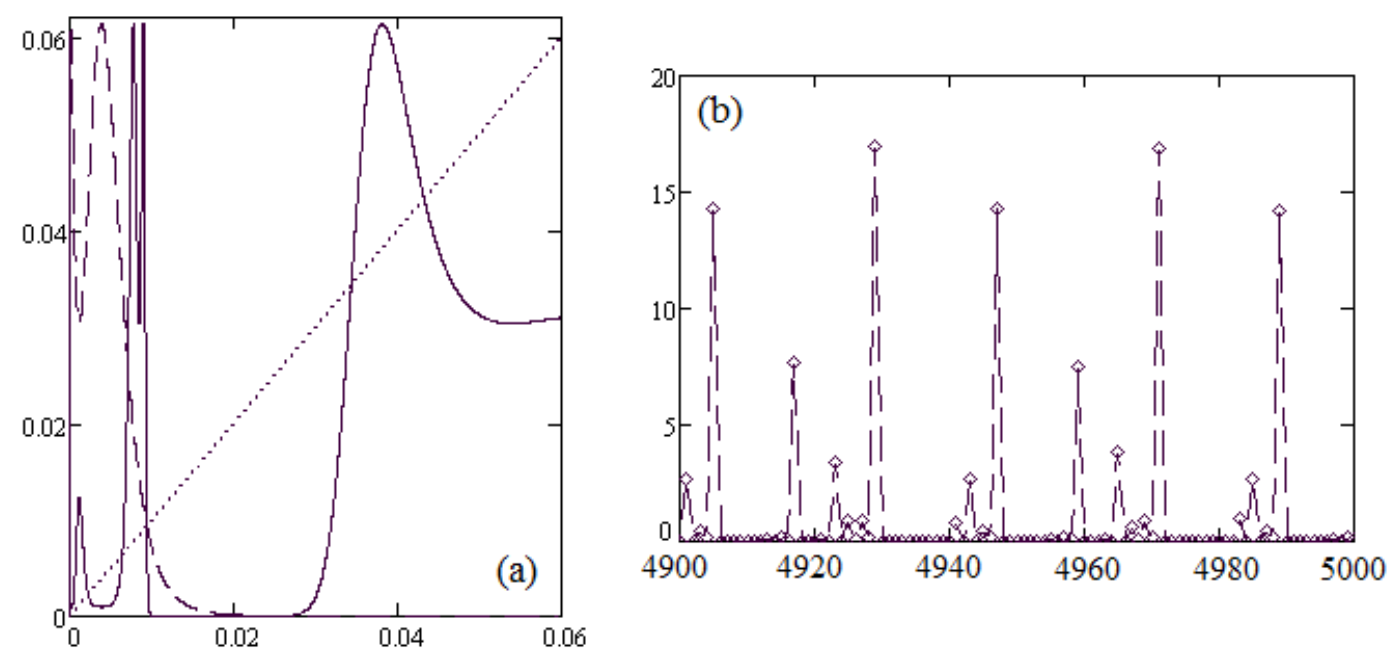

Figure 6: (a) Graphs of $f$ and $f^{3}$; (b) Corresponding nonperiodic solution 
Finally, in Figure 6 the initial values are $x_{-1}=1$ and $x_{0}=6$. Panel (a) shows the graphs of $f$ and $f^{3}$ where we can identify a pair of unstable 3 -cycles where the graph of $f^{3}$ crosses the identity line (in addition to the unstable fixed point of $f$ ). The map $f$ then exhibits Li-Yorke type chaos. A portion of the plot of the corresponding solution of (1) is shown in Panel (b). This nonperiodic solution coexists stably with the periodic solutions mentioned above. However, nonperiodic solutions do not exist under the hypotheses of Corollary 9 .

\section{The even period case}

When $\left\{a_{n}\right\}$ is periodic with minimal even period $p$ the next result shows that the sequence $\left\{t_{n}\right\}$ is not periodic with the exception of a boundary case. This causes a fundamental change in the dynamics of (1). Once again, the quantity $\sigma$ is defined by (9), i.e.

$$
\sigma=-a_{0}+a_{1}-a_{2}+\ldots+a_{p-1}
$$

Lemma 14 Suppose that $\left\{a_{n}\right\}$ is a sequence of real numbers with minimal even period $p \geq 2$ and let $\left\{t_{n}\right\}$ be a solution of (3). Then

$$
t_{n}=\left(t_{0} e^{d_{n} \sigma+\gamma_{n}}\right)^{(-1)^{n}}
$$

where the integer divisor $d_{n}=[n-n(\bmod p)] / p$ is uniquely defined by each $n$ and

$$
\gamma_{n}= \begin{cases}\sum_{j=1}^{n(\bmod p)}(-1)^{j} a_{j-1} & \text { if } n(\bmod p) \neq 0 \\ 0 & \text { if } n(\bmod p)=0\end{cases}
$$

The sequence $\left\{t_{n}\right\}$ is periodic with period $p$ iff $\sigma=0$, i.e.

$$
a_{0}+a_{2}+\cdots a_{p-2}=a_{1}+a_{3}+\cdots+a_{p-1} .
$$

Proof. Let $\left\{a_{0}, a_{1}, \ldots, a_{p-1}\right\}$ be a full cycle of $a_{n}$ with an even number of terms. Since $n=$ $p d_{n}+n(\bmod p)$ for $n \geq 1$, expand $s_{n}$ in $(8)$ to obtain

$$
s_{n}=d_{n} \sigma+\sum_{j=1}^{n(\bmod p)}(-1)^{j} a_{j-1}
$$

if $n(\bmod p) \neq 0$. If $p$ divides $n$ so that $n(\bmod p)=0$ then we assume that the sum is 0 and $s_{n}=d_{n} \sigma$. Thus $s_{n}=d_{n} \sigma+\gamma_{n}$ where $\gamma_{n}$ is as defined in (21).

The $\sigma$ terms have uniform signs in this case since there are an even number of terms in each full cycle of $a_{n}$. Now (7) yields

$$
t_{n}=t_{0}^{(-1)^{n}} e^{(-1)^{n} s_{n}}=t_{0}^{(-1)^{n}} e^{(-1)^{n}\left(d_{n} \sigma+\gamma_{n}\right)}
$$


which is the same as 20 .

Next, if $\sigma \neq 0$ then $d_{n} \sigma$ is unbounded as $n$ increases without bound so $\left\{t_{n}\right\}$ is not periodic. But if $\sigma=0$ then 20 reduces to

$$
t_{n}=\left(t_{0} e^{\gamma_{n}}\right)^{(-1)^{n}}
$$

Since the sequence $\gamma_{n}$ has period $p$, the expression on the right hand side of (23) has period $p$ with a full cycle

$$
t_{1}=\frac{e^{a_{0}}}{t_{0}}, t_{2}=t_{0} e^{-a_{0}+a_{1}}, t_{3}=\frac{e^{a_{0}-a_{1}+a_{2}}}{t_{0}}, \ldots, t_{p}=t_{0} e^{-a_{0}+a_{1}+\cdots+(-1)^{p} a_{p-1}}=t_{0} .
$$

By the preceding result,

$$
\begin{aligned}
t_{2 m} & =t_{0} e^{\gamma_{2 m}} e^{d_{2 m} \sigma} \quad \text { if } n=2 m \text { is even } \\
t_{2 m+1} & =\frac{1}{t_{0}} e^{-\gamma_{2 m+1}} e^{-d_{2 m+1} \sigma} \quad \text { if } n=2 m+1 \text { is odd }
\end{aligned}
$$

Suppose that $\sigma \neq 0$. If $\sigma>0$ then since $\lim _{n \rightarrow \infty} d_{n}=\infty$ it follows that $t_{2 m}$ is unbounded but $t_{2 m+1}$ converges to 0 , and the reverse is true if $\sigma<0$. Therefore,

$$
\begin{array}{lll}
\lim _{m \rightarrow \infty} t_{2 m}=\infty, & \lim _{m \rightarrow \infty} t_{2 m+1}=0, & \text { if } \sigma>0, \\
\lim _{m \rightarrow \infty} t_{2 m}=0, & \lim _{m \rightarrow \infty} t_{2 m+1}=\infty, & \text { if } \sigma<0 .
\end{array}
$$

Lemma 15 Suppose that $\left\{a_{n}\right\}$ is a sequence of real numbers with minimal even period $p \geq 2$ and let $\left\{x_{n}\right\}$ be a solution of (1) with initial values $x_{-1}, x_{0}>0$. Then $\lim _{n \rightarrow \infty} x_{2 n+1}=0$ if $\sigma>0$ and $\lim _{n \rightarrow \infty} x_{2 n}=0$ if $\sigma<0$.

Proof. Assume first that $\sigma>0$. Then by (24) $\lim _{n \rightarrow \infty} t_{2 n}=\infty$ so as in the proof of Lemma 4 $\lim _{n \rightarrow \infty} x_{2 n+1}=0$. If $\sigma<0$ then a similar argument using (25) yields $\lim _{n \rightarrow \infty} x_{2 n}=0$ to complete the proof.

Lemma 15 indicates that half of the terms of every solution $\left\{x_{n}\right\}$ of (1) converge to 0 in the even period case if $\sigma \neq 0$. We now consider what happens to the other half.

Lemma 16 Let $\left\{u_{n}\right\}$ be the solution of

$$
u_{n+1}=u_{n} e^{a_{2 n+1}-u_{n}}
$$

and $\left\{w_{n}\right\}$ be the solution of

$$
w_{n+1}=w_{n} e^{a_{2 n+2}-w_{n}} .
$$

(a) The sequence $\left\{x_{n}\right\}$ with $x_{2 n}=u_{n}$ and $x_{2 n+1}=0$ is a solution of (1).

(b) The sequence $\left\{x_{n}\right\}$ with $x_{2 n}=0$ and $x_{2 n+1}=w_{n}$ is a solution of (1). 
Proof. (a) Let $\left\{u_{n}\right\}$ be a solution to (26) from initial value $u_{0}>0$. If $x_{0}=u_{0}$ and $x_{1}=0$, then

$$
x_{2}=x_{0} e^{a_{1}-x_{0}-x_{1}}=u_{0} e^{a_{1}-u_{0}}=u_{1}
$$

and

$$
x_{3}=x_{1} e^{a_{2}-x_{2}-x_{1}}=0
$$

Inductively, if $x_{2 k}=u_{k}$ and $x_{2 k+1}=0$ for some $k \geq 1$ then

$$
x_{2 k+2}=x_{2 k} e^{a_{2 k+1}-x_{2 k}-x_{2 k+1}}=u_{k} e^{a_{2 k+1}-u_{k}}=u_{k+1}
$$

and

$$
x_{2 k+3}=x_{2 k+1} e^{a_{2 k+2}-x_{2 k+1}-a_{2 k+2}}=0
$$

which proves (a).

(b) Let $\left\{w_{n}\right\}$ be a solution to 27 from initial value $w_{0}>0$. If $x_{0}=0$ and $x_{1}=w_{0}$, then

$$
x_{2}=x_{0} e^{a_{1}-x_{0}-x_{1}}=0
$$

and

$$
x_{3}=x_{1} e^{a_{2}-x_{2}-x_{1}}=w_{0} e^{a_{2}-w_{0}}=w_{1}
$$

Inductively, if $x_{2 k}=0$ and $x_{2 k+1}=w_{k}$ for some $k \geq 1$ then

$$
x_{2 k+2}=x_{2 k} e^{a_{2 k+1}-x_{2 k}-x_{2 k+1}}=0
$$

and

$$
x_{2 k+3}=x_{2 k+1} e^{a_{2 k+2}-x_{2 k+1}-a_{2 k+2}}=w_{k} e^{a_{2 k+2}-w_{k}}=w_{k+1}
$$

which proves (b).

The next result is proved in $[8]$.

Lemma 17 Consider the first-order difference equation

$$
y_{n+1}=y_{n} e^{\alpha_{n}-y_{n}}
$$

where $\alpha_{n}$ is a sequence of real numbers with period q. If $0<\alpha_{n}<2$ then (28) has a globally asymptotically stable solution $\left\{y_{n}^{*}\right\}$ with period $q$ such that

$$
\sum_{i=1}^{q} y_{i}^{*}=\sum_{i=1}^{q} \alpha_{i} .
$$


Theorem 18 Let $\left\{a_{n}\right\}$ be periodic with minimal even period $p \geq 2$ and let $\sigma$ be as defined in (9).

(a) If $\sigma>0$ and $0<a_{2 k-1}<2$ for $k=1,2, \ldots p / 2$ then (1) has a globally attracting periodic solution $\left\{\bar{x}_{n}\right\}$ with period $p$ such that $\bar{x}_{2 n-1}=0$ and $\bar{x}_{2 n}$ is a sequence of period $p / 2$ satisfying the equality

$$
\sum_{i=1}^{p / 2} \bar{x}_{2 i-2}=\sum_{i=1}^{p / 2} a_{2 i-1}
$$

(b) If $\sigma<0$ and $0<a_{2 k-2}<2$ for $k=1,2, \ldots p / 2$ then (1) has a globally attracting periodic solution $\left\{\bar{x}_{n}\right\}$ with period $p$ such that $\bar{x}_{2 n}=0$ and $\bar{x}_{2 n-1}$ is a sequence of period $p / 2$ satisfying the equality

$$
\sum_{i=1}^{p / 2} \bar{x}_{2 i-1}=\sum_{i=1}^{p / 2} a_{2 i-2} .
$$

Proof. We prove part (a) and part (b) is demonstrated similarly. By Lemma 17 the equation in (26) has a periodic solution of period $p / 2$ given by $\left\{u_{i}^{*}\right\}$ with $0 \leq i \leq p / 2-1$. By Lemma 16, the sequence $\left\{u_{0}^{*}, 0, u_{1}^{*}, 0, \cdots, u_{p / 2-1}^{*}, 0\right\}$ is a $p$ periodic solution of 11 This means that $\bar{x}_{2 n-1}=0$ and $\bar{x}_{2 n}=u_{i}^{*}$ with

$$
\sum_{i=1}^{p / 2} \bar{x}_{2 i-2}=\sum_{i=1}^{p / 2} u_{i}^{*}=\sum_{i=1}^{p / 2} a_{2 i-1} .
$$

Let the even indexed terms of the solution $\left\{x_{n}\right\}$ be defined as in 15 and for each $n \geq 0$, define

$$
F_{n}(x)=x e^{\rho_{n}-x-\mu_{n} x e^{-x}}
$$

Then $F_{n}\left(u_{n}^{*}\right)=u_{n+1}^{*}$. Now observe that with $\xi_{n}=F_{n} \circ F_{n-1} \circ \cdots \circ F_{0}$

$$
F_{n}\left(y_{n}\right)=F_{n}\left(F_{n-1}\left(y_{n-1}\right)\right)=F_{n}\left(F_{n-1}\left(\cdots F_{0}\left(y_{0}\right)\right) \cdots\right)=\xi_{n}\left(y_{0}\right)
$$

Also note that

$$
\left|\xi_{n}^{\prime}\left(y_{0}\right)\right|=\left|\prod_{i=0}^{n} e^{\rho_{i}-y_{i}-\mu_{i} y_{i} e^{-y_{i}}}\left(1-\mu_{i} y_{i} e^{-y_{i}}\right)\left(1-y_{i}\right)\right|
$$

Since $\mu_{n} \rightarrow 0$, for sufficiently large $N, 0<\left(1-\mu_{n} y_{n} e^{-y_{n}}\right) \leq 1$ for $n \geq N$. Then there exists a constant $M>0$ so that

$$
\left|\prod_{i=0}^{n}\left(1-\mu_{i} y_{i} e^{-y_{i}}\right)\right| \leq\left|\prod_{i=0}^{N}\left(1-z_{i+1}\right)\right| \leq M
$$

Proceeding now as in the proof of Lemma 7 , if we let $m=[n / 2]$, we can find constants $K>0$ and $\delta \in(0,1)$ so that

$$
\left|\prod_{i=0}^{n}\left(1-y_{i}\right)\right| \leq K \delta^{m}
$$


Therefore,

$$
\left|\xi_{n}^{\prime}\left(y_{0}\right)\right|=\frac{y_{n+1}}{y_{0}}\left|\prod_{i=0}^{n}\left(1-\mu_{i} y_{i} e^{-y_{i}}\right)\left(1-y_{i}\right)\right| \leq \frac{\beta}{\alpha} K M \delta^{m}
$$

Finally,

$$
\left|y_{n+1}-u_{n+1}^{*}\right|=\left|F_{n}\left(y_{n}\right)-F_{n}\left(u_{n}^{*}\right)\right|=\left|\xi_{n}\left(y_{0}\right)-\xi_{n}\left(u_{0}^{*}\right)\right|=\left|\xi^{\prime}(w)\right|\left|y_{0}-u_{0}^{*}\right| \leq \frac{b}{\alpha} K M \delta^{m}\left|y_{0}-u_{0}^{*}\right| \rightarrow 0
$$

as $n \rightarrow \infty$ which completes the proof.

Remark 19 1. In Theorem $18\left(\right.$ a) the even-indexed terms $a_{2 k}$ are not restricted to $(0,2)$ as long as $\sigma>0$, i.e.

$$
a_{1}+a_{3}+\cdots+a_{p-1}>a_{0}+a_{2}+\cdots+a_{p-2}
$$

This imposes an upper bound $a_{2 k}<2(p / 2)=p$ for each $k$ but clealy some $a_{2 k}$ may exceed 2. Similarly, in (b) the odd-indexed terms are not restricted to (0,2) as long as $\sigma<0$.

2. Note that $2 p$ is not a minimal period for $\left\{\bar{x}_{n}\right\}$. For example, if $p=4$ with $a_{1}=a_{3}$ and $2 a_{1}>a_{0}+a_{2}$ (so that $\sigma>0$ ) then $\bar{x}_{2 n-1}$ satisfies (28) with constant $\rho_{n}$. In this case, Lemma 17 yields a globally asymptotically stable fixed point for (28), and thus a globally attracting period two solution for (1).

Figure 7 illustrates Theorem 18 with $p=4$ and

$$
a_{0}=1.4, \quad a_{1}=1.8, \quad a_{2}=1.6, \quad a_{3}=0.3
$$

In the boundary special case $\sigma=0$, the solutions of (1) have entirely different dynamics that resemble the odd period case. Indeed, the next result is similar to Theorem 13 .

Corollary 20 Suppose that $\left\{a_{n}\right\}$ is periodic with minimal even period $p$ and $\sigma=0$. Let $f$ be the interval map in Lemma 11 where $t_{0}>0$ is a fixed real number and $t_{k}$ is given by (7)-(8) for $k \geq 1$.

(a) If $s$ is a periodic point of $f$ with period $\omega$ then all solutions of (1) with initial values $x_{-1}=s$ and $x_{0}=t_{0} s e^{-s}$ (i.e. $\left(x_{-1}, x_{0}\right)$ is on the curve $g_{0}$ ) have period $p \omega$.

(b) If the map $f$ has a non-periodic point, then (1) has a non-periodic solution.

(c) If $f$ has a period-three point then (1) has periodic solutions of period pn for all positive integers $n$ as well as chaotic solutions in the sense of Li-Yorke [4].

Proof. By Lemma $14\left\{t_{n}\right\}$ has period $p$ so the application of Lemma 12 completes the proof.

If $p=2$ in the above corollary then $a_{0}=a_{1}$ (because $\sigma=0$ ) so the sequence $\left\{a_{n}\right\}$ is constant, i.e. it has mimimal period 1 not 2 . The behavior described in the corollary is indeed that which is observed for the constant parameter case; see [3] for a discussion of the stability of the variety of solutions mentioned above, which is of the same type as noted in Remark 10. 


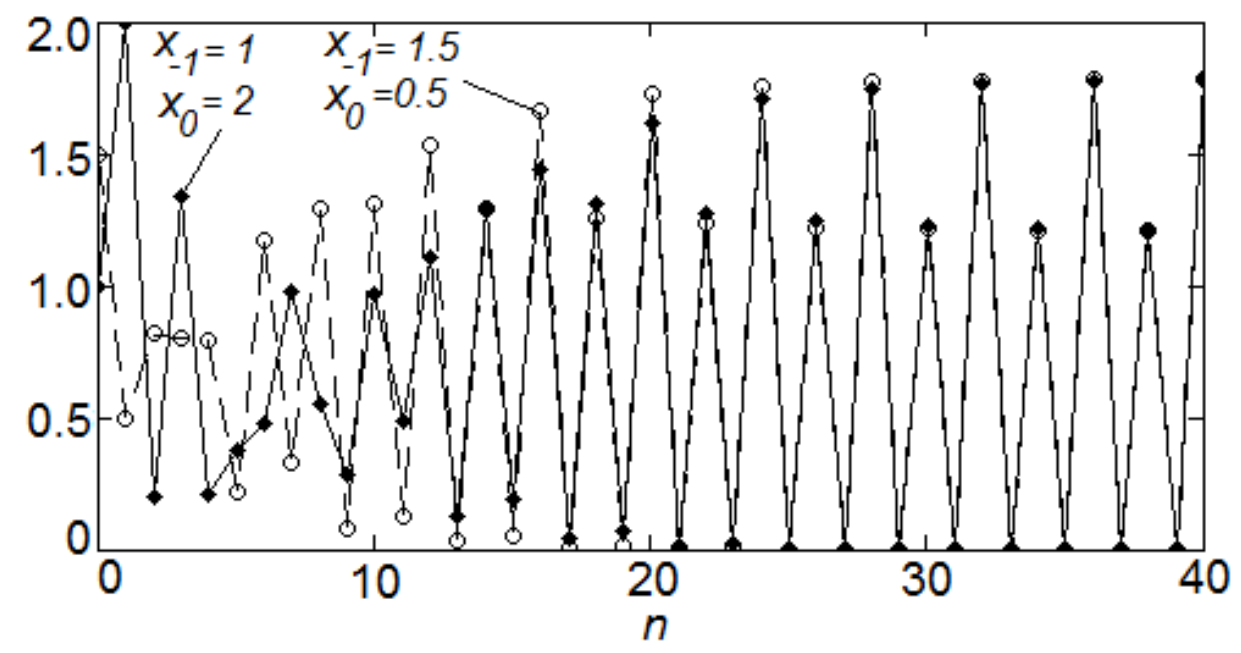

Figure 7: Solutions converging to a single 4-cycle with parameter period $\mathrm{p}=4$

\section{Summary and future directions}

We used a semiconjugate factorization of (1) to investigate its dynamics. Semiconjugate factorizations for difference equations of exponential type are not generally known (unlike linear equations) but fortunately we have one for (1). As we see above, the decomposition of (1) into the system (5)-(6) of first-order equations makes it clear why the solutions of (1) behave differently in a fundamental way depending on whether the period of $\left\{a_{n}\right\}$ is odd or even: in the former case the sequence $\left\{t_{n}\right\}$ is periodic, hence bounded while the latter case $\left\{t_{n}\right\}$ is unbounded when $\sigma \neq 0$.

The main results of this paper are Corollary 9 and Theorem 13 that discuss the dynamics of solutions if $p$ is odd and Theorem 18 if $p$ is even. Corollary 9 and Theorem 13 show that (1) has multistable coexisting solutions, including non-periodic and chaotic solutions if the amplitude of the parameter sequence $a_{n}$ is unrestricted. Theorem 18 indicates a completely different dynamics where globally stable limit cycles occur when $a_{n}$ is restricted to the interval $(0,2)$. Another of our main results is Theorem 8 that extends previous special cases in [2] and [3]. Further, Corollary 9 is a consequence of Theorem 8 .

An extension of Theorem 18 that includes non-periodic solutions when $a_{n}$ exceeds 2 for some indices $n$ is expected and may be of future interest. Such an extension evidently proves the existence of asymptotically stable non-periodic solutions, including chaotic solutions for (1) when $\sigma \neq 0$.

A natural extension of the above results is not obvious for higher order versions of (1) such as

$$
x_{n+1}=x_{n-1} e^{a_{n}-x_{n}-x_{n-k}}
$$

For instance, (29) may have unbounded solutions if $k \geq 2$ and exhibits different dynamics than (1). Further, known semicongjugate factorizations for (29) decompose it into a factor equation with 
order at least 2 if $k \geq 2$; see 9 . Such an equation is less tractable than the first-order case studied above. A detailed study of difference equations such as 29 and similar with periodic $\left\{a_{n}\right\}$ may yield interesting and possibly unexpected results.

\section{References}

[1] Elaydi, S.N., Discrete Chaos, 2nd ed., Chapman \& Hall/CRC, Boca Raton, 2008

[2] Franke, J.E., Hoag, J.T. and Ladas, G., Global attractivity and convergence to a two-cycle in a difference equation, J. Difference Eq. Appl. 5, 203-209, 1999

[3] Lazaryan, N. and Sedaghat, H., Extinction, periodicity and multistability in a Ricker model of Stage-structured populations, J. Difference Eq. Appl., 2016, DOI: 10.1080/10236198.2015.1123707

[4] Li, T-Y and Yorke, J.A., Period three implies chaos, Amer. Math. Monthly 82, 985-992, 1975

[5] Liz, E. and Pilarczyk, P., Global dynamics in a stage-structured discrete-time population model with harvesting, J. Theor. Biol. 297, 148-165, 2012

[6] Luis, R., Elaydi, S., Oliveira, H., Stability of a Ricker-type competition model and the competitive exclusion principle, J. Biol. Dyn. 5, 636-660, 2011

[7] Ricker, W.E., Stock and recruitment, Journal of Fish Research Board of Canada 11, 559-623, 1954

[8] Sacker, R., A Note on Periodic Ricker Maps. J. Difference Eq. and Appl., 13, 89-92, 2007

[9] Sedaghat, H., Form Symmetries and Reduction of Order in Difference Equations, CRC Press, Boca Raton, 2011

[10] Smital, J., Why it is important to understand the dynamics of triangular maps, J. Difference Eq. and Appl. 14, 597-606, 2008

[11] Zipkin, E.F., Kraft, C.E., Cooch, E.G., and Sullivan, P.J., When can efforts to control nuisance and invasive species backfire? Ecol. Appl. 19, 1585-1595, 2009. 